\title{
The dynamics of striatum circuitry
}

\author{
Shih-Chieh Lin* \\ Neural Circuits and Cognition Unit, Laboratory of Experimental Gerontology, National Institute on Aging, US National Institutes of Health, Baltimore, MD, USA \\ *Correspondence: shih-chieh.lin@nih.gov
}

\section{A commentary on}

Dynamic reorganization of striatal circuits during the acquisition and consolidation of a skill. by Henry H. Yin, Shweta Prasad Mulcare, Monica R.F. Hilário, Emily Clouse, Terrell Holloway, Margaret I. Davis, Anita C. Hansson, David M. Lovinger and Rui M. Costa (2009). Nat. Neurosci. 12, 333-341.

Learning new motor skills is an integral and essential part of our life. Remember the first time riding a bike or learning to ski? After a new skill is acquired, the once daunting task becomes effortless and automatic. How does our brain accomplish such a feat? Which brain systems are involved, and how? A recent article by Yin et al. (2009) provides novel mechanistic insights on this issue.

Behavioral evidence indicates that motor skill learning takes place over an initial phase of fast improvements, followed by a slower phase of gradual refinement (Karni et al., 1998; Costa et al., 2004). An interesting parallel that similarly consists of a fast and slow learning phase is reward-guided instrumental learning (Yin and Knowlton, 2006; Yin et al., 2008). The initial phase of rewardguided instrumental learning is deliberate and sensitive to both the action-outcome contingency and the value of the outcome. Prolonged training transforms an action into a habit, such that the once deliberate action becomes automatic and insensitive to both the action-outcome contingency and to the outcome value.

Recently, the roles of different striatal subregions in reward-guided instrumental learning have been extensively investigated. Two subregions of the striatum, specifically the dorsomedial striatum (DMS) and the dorsolateral striatum (DLS), have been shown to play crucial roles in the learning of goal-directed actions and habit, respectively. Lesions and pharmacological inactivation of the DMS (DLS) impairs the acquisition of goal-directed actions (habit) (Yin et al., 2005, 2006). Such roles are consistent with their anatomical connection patterns, with the DMS receiving inputs from the prefrontal cortex and the DLS receiving inputs from sensorimotor cortices.

Since the striatum is also essential for motor skill learning, the findings that the DMS and DLS are important for rewardguided instrumental learning raises the question whether the DMS and DLS play similar roles in the fast and slow phases of motor skill learning. This question is what Yin et al. set out to address. Using a rotarod task, the authors found that lesions of the DMS impaired skill learning only in the early phase, similar to the effect of DMS lesions in reward-guided instrumental learning. Moreover, with simultaneous in vivo extracellular recording in the DMS and DLS of the same animal, the authors showed that the activity of DMS neurons is preferentially modulated during the early phase of rotarod learning, while DLS neurons show modulation only in the late phase. This is the first direct neurophysiological evidence that the DMS and DLS play a dynamic and perhaps sequential role in skill learning.

After demonstrating the regionspecificity of skill learning, Yin et al. further provided detailed circuit level mechanisms underlying the dynamic reorganization of the memory trace in striatal circuits. Taking brain slices from adult mice that are either naïve, or during early or late phases of rotarod learning, the authors demonstrated that the differential engagement of DMS and DLS neurons in early and late phases of skill learning, respectively, likely resulted from the potentiation of glutamatergic synapses onto medium spiny neurons, but not from a learning-related change of intrinsic excitability in medium spiny neurons. Furthermore, using D2-enhanced GFP (EGFP) mice to visualize striatopallidal medium spiny neurons, i.e., 'indirect' pathway, Yin et al. was able to show that the enhanced synaptic strength in the DLS after extended training is mostly restricted to the striatopallidal neurons expressing the D2-receptor, but not in neighboring striatonigral D1-expressing medium spiny neurons. This pathway-specific engagement is supported by pharmacological manipulations: while both D1- and D2-receptor blockades impaired rotarod performance in naïve and early phase mice, the performance after extended training is sensitive only to D2-receptor blockade.

These findings highlight the dynamic nature of motor skill learning in the striatal circuitry, with the DMS engaged early in the acquisition phase and a subsequent shift over to the DLS later in the consolidation phase. The roles of the DMS and DLS in motor skill learning are likely not strictly sequential, but rather competing for the control of a behavior in a dynamic fashion. For example, a habitual behavior typically under the control of the DLS can become sensitive to outcome values and thus goaldirected when the DLS is inactivated or removed (Yin et al., 2006), suggesting that the DMS can gain control of this type of behavior when the DLS is not involved. The results from Yin et al. provide an important initial step in visualizing this dynamic competition between parallel neural circuits, which is perhaps a property of neural systems in general. The challenge going forward is to understand how the DMS and DLS compete for control of a behavior, not only over the course of learning but also in real-time on a trial-by-trial basis. Is there a neurophysiological signature that can reveal the dominant neural systems/pathways at any given moment?

Overall, Yin et al. provided an elegant example in dissecting circuit mechanisms of behavior through behavioral testing, in vivo multi-site extracellular recording, ex vivo slice physiology studies and mouse genetics tools. This combination of converging approaches leads to novel insights on the circuit mechanisms of motor skill learning with single neuron resolution and pathway-specificity, and serve as a model to tackle circuit-level mechanisms in other neural systems. 


\section{ACKNOWLEDGMENTS}

The author likes to thank Geoff Nelson for comments and discussions. This research was supported entirely by the Intramural Research Program of the NIH, National Institute on Aging.

\section{REFERENCES}

Costa, R. M., Cohen, D., and Nicolelis, M. A. (2004). Differential corticostriatal plasticity during fast and slow motor skill learning in mice. Curr. Biol. 14, 1124-1134.

Karni, A., Meyer, G., Rey-Hipolito, C., Jezzard, P., Adams, M. M., Turner, R., and Ungerleider, L. G. (1998). The acquisition of skilled motor performance: fast and slow experience-driven changes in primary motor cortex. Proc. Natl. Acad. Sci. U.S.A. 95, 861-868.

Yin, H. H., and Knowlton B. J. (2006). The role of the basal ganglia in habit formation. Nat. Rev. Neurosci. 7, 464-476.

Yin, H. H., Knowlton, B. J., and Balleine, B. W. (2006). Inactivation of dorsolateral striatum enhances sensitivity to changes in the action-outcome contingency in instrumental conditioning. Behav. Brain Res. 166, 189-196.

Yin, H. H., Mulcare, S. P., Hilario, M. R., Clouse, E., Holloway, T., Davis, M. I., Hansson, A. C., Lovinger, D. M., and Costa, R. M. (2009). Dynamic reorganization of striatal circuits during the acquisition and consolidation of a skill. Nat. Neurosci. 12,333-341.

Yin, H. H., Ostlund, S. B., Knowlton, B. J., and Balleine, B. W. (2005). The role of the dorsomedial striatum in instrumental conditioning. Eur. J. Neurosci. 22, 513-523.

Yin, H. H., Ostlund, S. B., Knowlton, B. J., and Balleine, B. $\mathrm{W}$. (2008). Reward-guided learning beyond dopamine in the nucleus accumbens: the integrative functions of cortico-basal ganglia networks. Eur. J. Neurosci. 28, 1437-1448.

Received: 20 January 2010; published online: 09 February 2010.

Citation: Lin S-C (2010) The dynamics of striatum circuitry. Front. Integr. Neurosci. 4:3. doi: 10.3389/neuro.07.003.2010 Copyright $\odot 2010$ Lin. This is an open-access article subject to an exclusive license agreement between the authors and the Frontiers Research Foundation, which permits unrestricted use, distribution, and reproduction in any medium, provided the original authors and source are credited. 